\title{
Nasal fibrosis: long-term follow up of four cases of eosinophilic angiocentric fibrosis
}

\author{
Santdeep Paun, FRCS (ORL-HNS), VALerie J Lund, MS, FRCS, FRCS(Ed), \\ ANDREW GALLIMORE, BSC, MRCP, FRCPATH
}

\begin{abstract}
Eosinophilic angiocentric fibrosis is a rare, benign cause of submucosal thickening and fibrosis within the upper respiratory tract. It predominantly affects the nose although cases have been reported in the subglottis. We describe four cases of the disease centred around the nasal cavity, with widespread infiltration of the facial soft tissues and orbit in three of the four patients. Each underwent long term follow up. Multiple surgical resections were required with two of our patients and, to date, medical therapy has been of limited help. The disease process, with its clinical and characteristic histopathological findings, is described. We also discuss the management of the disease following a comprehensive review of, and comparison with, the few prior reported cases.
\end{abstract}

Key words: Eosinophilic Angiocentric Fibrosis; Upper Respiratory Tract; Nasal Septum; Stenosis

\section{Introduction}

Eosinophilic angiocentric fibrosis (EAF) remains an unusual, benign condition of the upper respiratory tract. Since the first description in $1985,{ }^{1}$ some further cases have been published. ${ }^{2-8}$ The aetiology of the condition remains unknown, but clinically there is typically submucosal thickening with subsequent fibrosis largely within the nasal cavity, although two cases have been reported within the subglottis. The nasal septum appears to be most commonly affected although extension to the lateral wall has been seen. The condition is slow-growing and until recently has proved a diagnostic difficulty, with prior histological reports giving no specific cause for the inflammation and fibrosis evident. The specific histological features include a perivascular inflammatory cell infiltration with progressive fibrosis around small vessels, leading to a characteristic whorling, 'onion-skin'-type pattern. ${ }^{1}$ Eosinophils represent the predominant inflammatory cells, particularly with progression of the fibrosis. The histological appearance closely resembles that of the equally rare skin lesion granuloma faciale and is likely to represent a mucosal variant thereof.

We report a further four cases of EAF centred around the nasal cavity and describe their management and long term follow up. We review the limited pertinent literature currently available for this rare condition and make comparisons between our own and previously published cases.

\section{Case studies}

\section{Case one}

A 37-year-old woman presented as a tertiary referral to our department in July 1995. She had originally been seen and treated in Germany for some 18 years prior to relocating to the United Kingdom. Her first presentation was at age 19 when she had developed a small skin lesion in the left frontonasal region which appeared to extend into the nasal cavity. This was subsequently biopsied, initially endonasally, and a chronic inflammatory infiltrate was identified, but no specific histological diagnosis could be ascertained. The lesion thereafter grew dramatically and a further external biopsy was performed some months later. Histological diagnosis at this time pointed towards an early form of scleroma and no specific malignant features could be identified. A lateral rhinotomy was performed at this stage with removal of the mass, inferior turbinate and a large amount of mucosa. Definitive histology once again was nonspecific although frozen sections had pointed to the possibility of mesenchymal malignancy. Further biopsies and a subsequent repeat lateral rhinotomy for formation of an endonasal chocolate cyst confirmed nonspecific perivascular round-cell inflammation.

Upon presentation to our care the patient had been resident in the UK for a year. She had developed thickening in the region of the frontal process and infraorbital margin of the left maxillary 


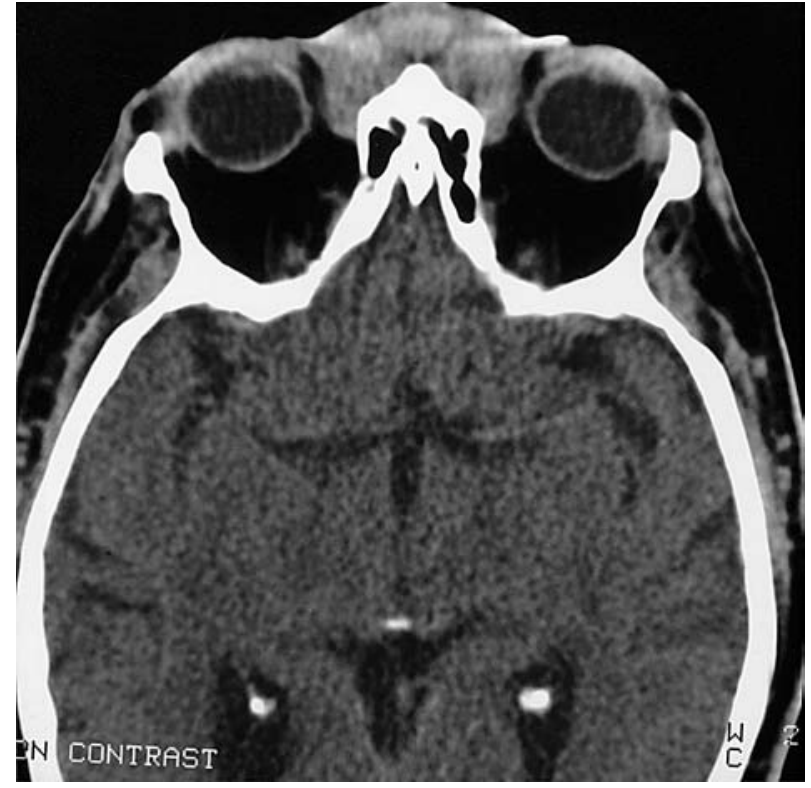

(a)

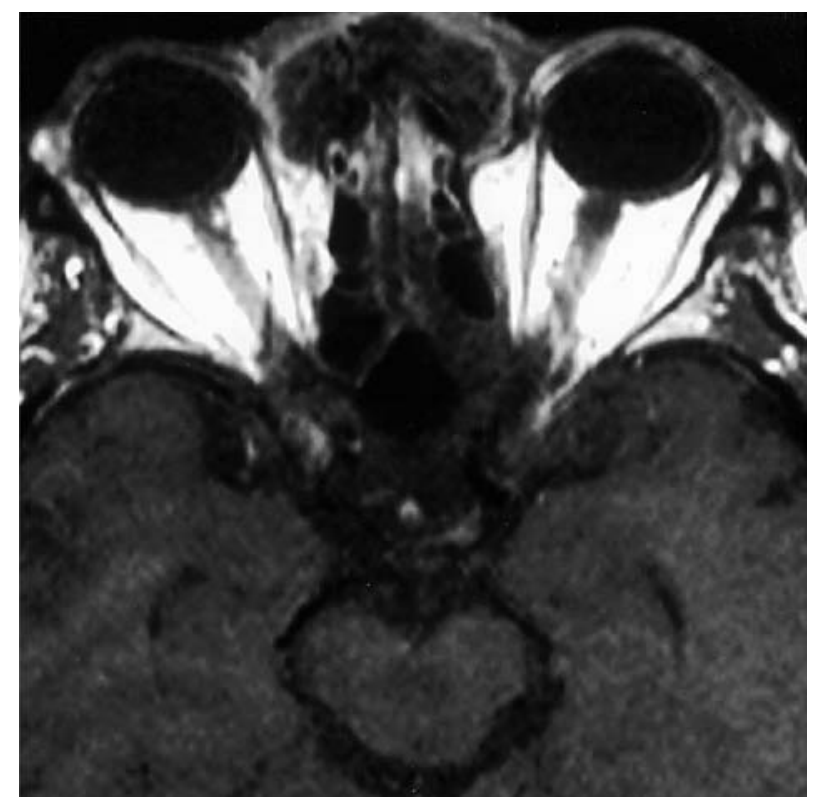

(b)

FIG. 1

(a) Axial CT showing mass in glabella region extending into orbital periosteum bilaterally. (b) Axial MRI (T1-weighted with gadolinium) of the mass in glabella region extending into orbital periosteum bilaterally.

bone together with a deeply tethered, poorly healed lateral rhinotomy scar. Her main complaints were of crusting, perimenstrual epistaxis, epiphora and symptoms of eustachian tube dysfunction. She was penicillin-sensitive but otherwise denied any allergies. Routine haematological screening, including erythrocyte sedimentation rate (ESR) and Anti-neutrophil cytoplasmic antibody (ANCA) assays, was unremarkable.

The lateral rhinotomy was reopened and a subdermal fibrous mass dissected out which appeared to be contiguous with the orbital periosteum which was resected. Inspection of the maxillary sinus showed some polypoidal mucosal changes although the ethmoid cavity was clear. A left grommet was also inserted for a serous effusion. Histological analysis confirmed features consistent with granuloma faciale and its intranasal variant, eosinophilic angiocentric fibrosis.

The patient remained well thereafter, with no evidence of recurrence of the mass on follow up, until some three years later when she presented with a further nodular, discoloured area over the left nasal bridge. An axial computed tomography (CT) scan confirmed a soft tissue mass encroaching on the orbit in the region of the medial canthus. To avoid further surgical resection, with its concomitant reconstruction difficulties, the patient was referred to a specialist dermatologist who commenced her on oral steroids and azathioprine. Her lesion improved considerably with this treatment although she had to discontinue it due to intolerance of side effects.

Over the next three years there was gradual progression of the lesion and the patient was re-referred, three months prior to the time of writing, with obvious bilateral infiltration of the nasal bridge extending into the glabella region, with a violaceous lesion of the nasal skin. Computed tomography and magnetic resonance imaging (MRI) scans confirmed that the lesion extended into the soft tissues of the contralateral medial canthal region and affected the left anterior orbit. A radical clearance of the area was undertaken through an extended lateral rhinotomy with sacrifice of the affected overlying skin, underlying cartilage and resection of the intraorbital mass. The defect was repaired with a median glabella flap together with skin grafting to the exposed orbital fat.

\section{Case two}

A 68-year-old gentleman presented as a tertiary referral to our care with a longstanding deformity in the nasal bridge and forehead region. It appeared to have gradually increased in size over some five years. He had a degree of nasal blockage but was otherwise well, with no other rhinological symptoms and no history of allergies. On examination, other than the swelling, there was hypertelorism, although ophthalmological assessment was otherwise normal. Intranasally, the disease process extended into the nasal septum, which was thickened and widened thus causing the obstruction. Radiologically, there appeared to be a large mass in the glabella region extending over the bridge of the nose and up to and including the upper lateral cartilages. The mass extended into both orbits, involving the orbital periosteum (Figure 1). Haematological testing once again showed no abnormality. Prior to referral, initial biopsies had revealed no specific diagnosis, but areas of chronic inflammatory infiltration were clearly seen within the otherwise benign process. The lesion was then further explored under our care through an extended lateral rhinotomy incision. The mass was extensively resected over the frontal and nasal bones, including a cuff of orbital periosteum, until it was felt a complete clearance had been achieved. The structural aspect of the nasal septum was left 


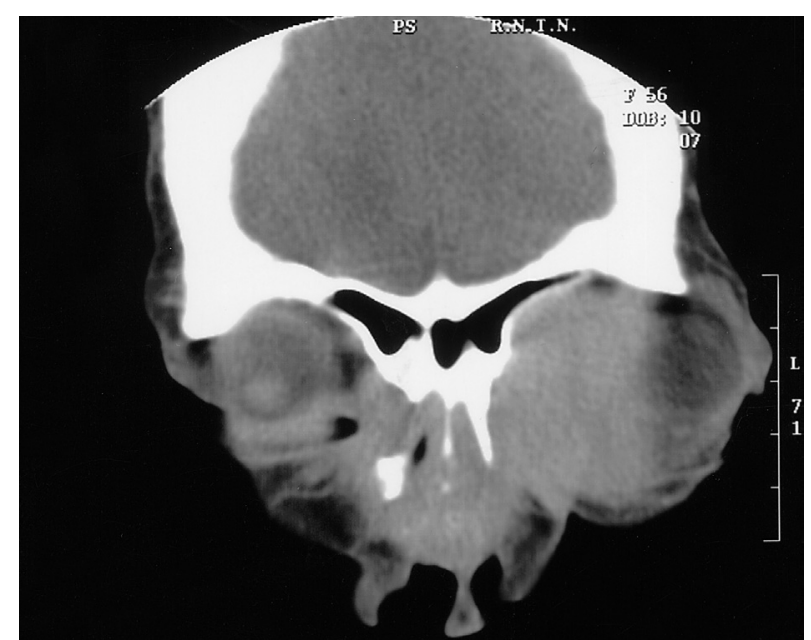

(a)

\section{FIG. 2}

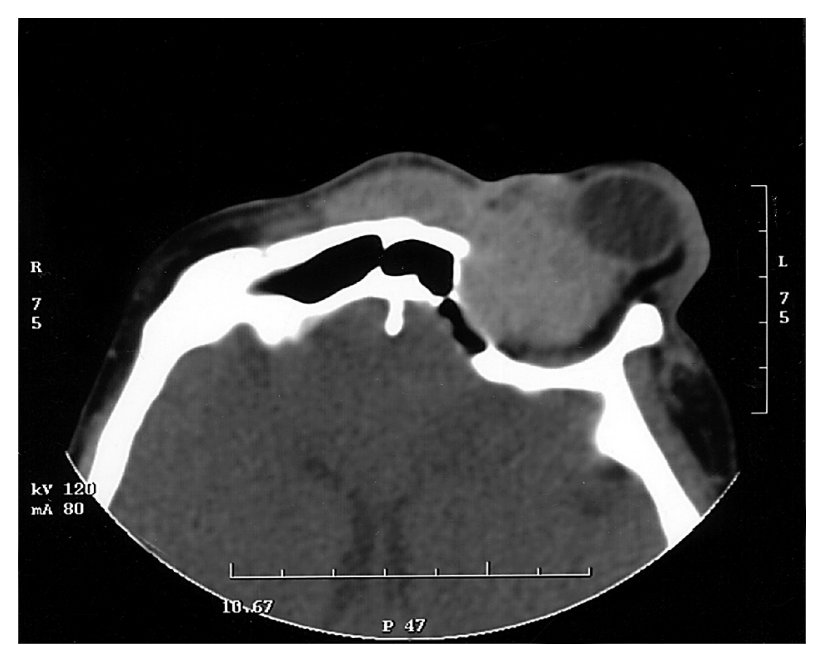

(b)

(a) Coronal CT showing massive infiltration of left orbit with lesion crossing midline to contralateral medial canthal region. (b) Axial CT showing soft tissue mass extending bilaterally from glabella region with wide infiltration of left orbit.

largely intact for support, although a portion of submucoperiosteal fibrosis was resected for diagnostic purposes. Histological evaluation of this large mass of tissue confirmed the characteristic findings associated with eosinophilic angiocentric fibrosis. Post-operatively, and over long term follow up of some six years, the patient remained well with no evidence of return of the mass and minimal nasal symptoms, although the nasal septum was noted to remain rather wide.

\section{Case three}

A 57-year-old Indian lady was originally referred to our department some 20 years ago with a nasal mass affecting both the internal and external structure of the nose, with extension into the maxillary cavity. The left nasal cavity was noted to be narrowed by an infiltrating mass which had also caused bony enlargement around the maxilla. The patient had had a prior lateral rhinotomy and went on to have a further such procedure to remove recurrent disease. At the time, a tuberculous mass, rhinoscleroma and fungal infection were thought to be the likely differential diagnoses, but each was excluded histologically. Fibrosis with infiltration of eosinophils was noted. Further biopsies confirmed a similar picture, with no granulomatous disease present, and serial cANCA and angiotensin-converting enzyme (ACE) levels were normal. It was only on a subsequent further lateral rhinotomy some years later that the diagnosis of eosinophilic angiocentric fibrosis was confirmed. The disease at the time had progressed significantly to occupy the anterior cheek, extending onto the nasal bridge, glabella region and into the orbit as well as causing swelling of the nasal septum and vestibular stenosis. Bone was noted to be dehiscent in the anterior aspect of the maxilla. Whilst the mass surrounding the orbit was extensively removed, the tissue extending into the orbit was left untouched at the patient's request, due to the obvious risks to vision. In an attempt to prevent progression of disease, particularly into the orbital tissues, the patient was referred for a specialist dermatological opinion regarding medical treatment. Over some years, treatment with dapsone, hydroxychloroquine and azathioprine was variously tried but proved to be less than successful, with no obvious shrinkage of the mass and no inhibition of recurrence. Oral steroids were briefly given and produced some reduction in the mass, but the patient was reluctant to continue with these in the longer term. Pulsed high-dose parenteral steroid treatment was also suggested, but again the patient would not undergo such treatment.

At the time of writing the disease had progressed significantly, with widespread infiltration of the left orbit and midfacial tissues and extension across the midline into the right medial canthus region (Figure 2 ). There was significant proptosis of the left eye, with reduction in vision. The patient refused further surgical intervention despite numerous attempts to warn her of the progressive nature of the disease.

\section{Case four}

Our fourth case represents a less extensive variant of the condition. A 58-year-old lady initially presented with a one-year history of a firm mass occupying the left lateral wall of the nose. The mass was removed via a lateral rhinotomy and was found to extend from the nasal bone down to the vestibule, involving the floor of the nose but not the turbinates. The patient had an uneventful recovery and at the time of writing, some three years on, there was no recurrence of the mass. Histopathological analysis once again was not specific at the referring hospital but the definitive diagnosis was made after referral to our department; the typical features of a fibrous lesion with a whorled, nodular architecture and scattered eosinophils were noted.

\section{Histopathological findings}

In their description of the histological findings, Roberts and McCann ${ }^{1}$ reported consistent features in the biopsy specimens of their three patients. An early 


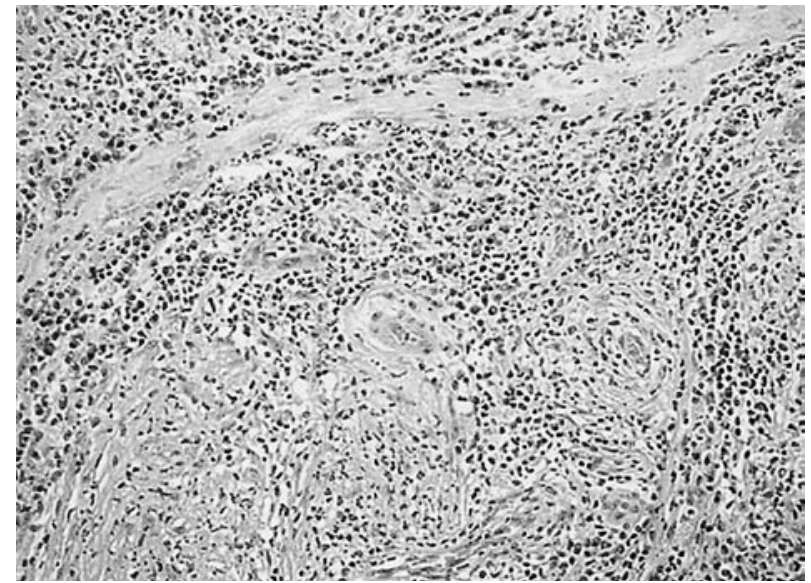

FIG. 3

Venule shows concentric infiltration by polymorphic inflammatory cell infiltrate, rich in eosinophils (H \& E; × 10).

eosinophil-rich vasculitic picture emerged centred around smaller blood vessels. No thrombosis or fibrinoid necrosis was found but the small vessel endothelium appeared swollen and leucocytoclasis featured. Whilst a granulomatous-type picture was present, the absence of multinucleated giant cells or epithelioid histiocytes excluded true granulomas. Further reports ${ }^{3-8}$ confirmed this picture of a perivascular inflammatory response involving capillaries and venules but not other vessels. Whilst eosinophils predominate, lymphocytes and plasma cells are also present. Accompanying this picture, in adjacent areas mural thickening in an 'onion skin' pattern appears. This intimal fibrosis, with characteristic whorling of the collagen fibres and reticulin, is thought to represent a later stage of the disease process. Eosinophils begin to predominate further as the other inflammatory cells progressively reduce in number. The histological findings in our cases are consistent with this picture (Figures 3 to 5), and whilst in case one we were unable to obtain the original histopathological slides from the patient's initial biopsies in Germany, we did receive pathology reports which were in keeping with the diagnosis, together with confirmation from subsequent histology.

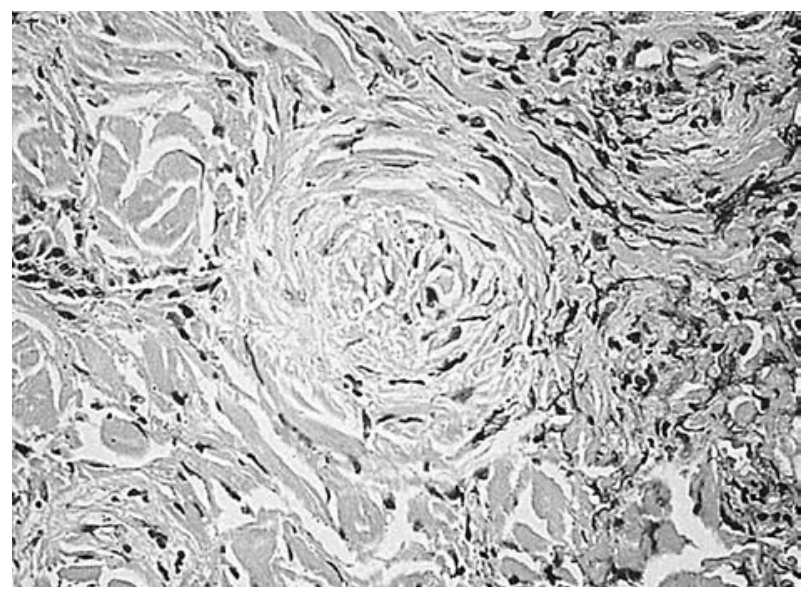

FIG. 5

Concentric fibrosis around central vessel $(\mathrm{H} \& \mathrm{E} ; \times 10)$.

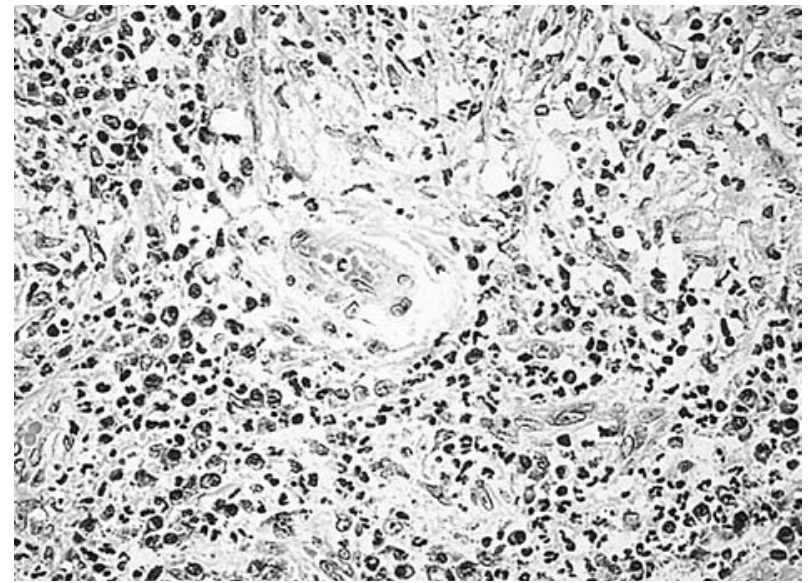

FIG. 4

Polymorphic perivascular inflammatory cell infiltrate, including lymphocytes, plasma cells and eosinophils (H \& E; ×20).

\section{Discussion}

The absence of any clear aetiology for this rare condition certainly warrants reporting of cases to enable further study into the disease process. It appears clear that, to date, cases may have gone undiagnosed, and pathological reports of nonspecific chronic inflammation/fibrosis of nasal biopsies may require further evaluation, particularly in the presence of what appears to be a progressive inflammatory process. Eosinophilic angiocentric fibrosis is a clear descriptive term applied to the biopsies of three patients by Roberts and McCann, ${ }^{1,2}$ although an earlier description of an intranasal mass arising in a 49-year-old man with typical features of granuloma faciale appears to be the first report of this condition. ${ }^{9}$ Fourteen further cases have been reported in the literature, the majority within the nasal cavity although the subglottis has also been involved in two cases. ${ }^{1,3}$ Both of these patients required laryngotracheoplasty following breathing difficulties. Histologically, EAF resembles granuloma faciale closely, with both conditions having a vasculitic appearance with prominent eosinophilic infiltration. The latter stages show an angiocentric fibrotic pattern although it is not so marked in granolma faciale. The relationship of EAF to granuloma faciale is still under some debate although, in the presence of such histological similarity and the coexistence of both lesions in many of the reported cases, it seems likely that EAF is a submucosal variant affecting the upper respiratory tract.

Table 1 gives a summary of the reported EAF cases presenting within the nasal cavity to date. Clearly, there appears at this stage to be a female preponderance, which is somewhat unusual considering granuloma faciale is more common in men. If EAF indeed represents a variant of granuloma faciale, it is not entirely clear why this inherent difference is seen. The nasal septum appears to be involved in most cases, and when considering causes for a septal thickening EAF should be entertained as an uncommon, but definite, differential diagnosis. The lateral wall of the nose may also be 
TABLE I

REPORTED CASES OF EOSINOPHILIC ANGIOCENTRIC FIBROSIS WITHIN THE NASAL CAVITY

\begin{tabular}{|c|c|c|c|c|}
\hline Report & Age/sex & Presenting lesion & Management & Long term follow up \\
\hline Holmes \& Panje ${ }^{9}$ & 49 Female & Intranasal mass & Surgical excision & Unknown \\
\hline \multirow[t]{4}{*}{ Roberts \& McCann ${ }^{1}$} & 27 Female & Septal/turbinate thickening & Surgical excisions & Residual disease \\
\hline & 59 Female & $\begin{array}{l}\text { Septal thickening/mass } \\
\text { over nasal bridge }\end{array}$ & Surgical excisions & Residual disease \\
\hline & 54 Female & Septal thickening & $\begin{array}{l}\text { Surgical excisions/ } \\
\text { radiotherapy }\end{array}$ & Unavailable \\
\hline & 50 Female & $\begin{array}{l}\text { Submucosal mass over } \\
\text { alar cartilage }\end{array}$ & Surgical excisions & Residual disease \\
\hline Altemani et al. ${ }^{5}$ & 54 Female & Septal/lateral wall thickening & Surgical excisions & Residual disease \\
\hline Matai et al. & 51 Male & Septal thickening & Surgical excision & Residual disease \\
\hline \multirow[t]{3}{*}{ Thompson \& Heffner ${ }^{8}$} & 28 Male & Septal/maxillary sinus mass & $\begin{array}{l}\text { Surgical excision/ } \\
\text { steroids }\end{array}$ & Residual disease \\
\hline & 49 Female & Septal thickening & $\begin{array}{l}\text { Surgical excision/ } \\
\text { steroids }\end{array}$ & Residual disease \\
\hline & 64 Female & Septal/maxillary sinus mass & $\begin{array}{l}\text { Surgical excision/ } \\
\text { steroids }\end{array}$ & Residual disease \\
\hline Burns et al. ${ }^{6}$ & 38 Male & $\begin{array}{l}\text { Septal thickening/mass } \\
\text { from alar cartilage }\end{array}$ & Surgical excisions & Residual disease \\
\hline Loane et al. ${ }^{4}$ & 42 Male & $\begin{array}{l}\text { Septal deviation/nasal } \\
\text { adhesions post-septoplasty }\end{array}$ & Surgical excision & Unavailable \\
\hline Pereira et al. ${ }^{10}$ & 52 Male & Septum & Surgical excisions & Unavailable \\
\hline Tabaee et al. ${ }^{11}$ & 79 Male & Septum and lateral wall & Surgical resection & Unavailable \\
\hline Nguyen et al. $^{12}$ & 45 Male & Septum/middle meatus & Surgical excision & Unknown \\
\hline
\end{tabular}

affected and this may clinically be used to exclude the midline granulomatous disease differentials. Wegener's granulomatosis was excluded by cANCA assays in all cases except that reported by Loane et al. ${ }^{4}$ These authors reported a possible coexistence of EAF in a patient with known Wegener's granulomatosis that had been treated successfully with immunosupression therapy. The report speculated that EAF may represent an exaggerated fibrosis of the upper airway rather than a distinct clinical entity. Our patients' histologies did not show these appearances, but were rather more in keeping with the original description of this condition, with no evidence of granulomatous disease, and this indeed appears to be the case in all the other cases thus far reported. As would be expected by the submucosal nature of the disease process, ulceration has not been a feature in any of the cases.

An allergic basis to the condition was suggested in early reports, based on specific allergies suffered by the reported patients and the abundant eosinophilic presence. There appears to be little definite correlation, however, with allergic history in subsequent published cases and it would seem unlikely that this represents an aetiological factor for the disease. In our series one of the patients had a penicillin sensitivity, with no other allergies reported.

Reviewing the prior literature, little comment has been made regarding the radiological findings and indeed it appears many of the cases were not imaged as the presenting feature was related to the nasal septum alone. Of those cases reported that mention radiology, it appears that plain sinus X-rays showed no abnormality ${ }^{1}$ and CT findings showed nonspecific soft-tissue swelling of the septum and lateral walls with no true bony destruction. ${ }^{6}$ In our series bony destruction was clearly a feature, with the disease process affecting the orbital periosteum. Depending on the extent of disease, both CT and MRI imaging is likely to be of benefit in assessment.
Three of our cases would appear to be rather extensive examples of the disease, which extended from the intranasal cavity into the paranasal sinus and infiltrated the orbital contents. In all of our cases the disease process was slow-growing, and in two of our cases disease was recurrent despite several radical attempts at surgical clearance. The length of time for disease progression reflects the slow, indolent nature of the disease process, and the rarity of the condition and the scarcity of previously published literature also result in delay in diagnosis. With growing awareness of the condition, earlier histological diagnosis may assist in appropriate management strategies and even more radical surgery $a b$ initio; the two cases in which this was undertaken in the first instance had no recurrence at the time of writing.

Surgical resection appears to be the treatment option of choice although recurrences are common in most prior published cases and multiple excisions are frequently required. Steroid therapy has had limited reported success, although we did find that two of our patients responded somewhat to this and to cytotoxic treatment, but unfortunately could not tolerate the side effects. The use of dapsone has been reported in granuloma faciale ${ }^{13,14}$ but unfortunately did not prove to be successful in the one patient for whom it was prescribed in our series. Hydroxychloroquine, seen to be of occasional benefit in vasculitic disorders, was also tried but was not helpful. The use of pulsed methylprednisolone was suggested as a possible treatment for our third patient, based on experiences of this approach for thyrotoxic-induced severe exophthalmos. ${ }^{15}$ This therapy was refused by our patient due to anxiety about steroid toxicity; however, it remains a possible treatment option for patients with progressive disease unsuitable for, or unwilling to undergo, surgery. 


\section{Conclusion}

Our long term follow up of these four patients appears to show the slow-growing but in some cases progressive nature of eosinophilic angiocentric fibrosis. The nature of this disease remains unknown, but as more cases are reported in the literature the natural history of this condition is becoming clearer. The nasal septum is most commonly affected although the lateral wall may also be involved. Soft tissue swelling around the midface may also be a presenting feature and subsequent infiltration into the orbital structures appears to be inevitable if the disease progresses, despite surgical clearances. Surgical resection of the disease remains the main treatment although multiple procedures are often required. Short term steroid treatment proved to be of some benefit in two of our more difficult patients, but long term use and, indeed, pulsed high-dose treatment require further evaluation. The characteristic histological findings of EAF are now well established and, with increasing awareness amongst histopathological colleagues, further reports of this rare condition should be produced.

- Eosinophilic angiocentric fibrosis is an idiopathic condition presenting with submucosal thickening and subsequent fibrosis that is seen most commonly in the nasal septum

- This is a paper reporting four cases. In three there was widespread infiltration of the facial soft tissue and orbit

- The disease process and the management of these patients is presented

\section{Acknowledgements}

We would like to thank $\mathrm{Mr}$ Andrew Grace for referring and helping manage two of the patients, Professor Roderick Hay for his dermatological input and Mr Geoffrey Rose for the ophthalmological care provided.

\section{References}

1 Roberts PF, McCann BG. Eosinophilic angiocentric fibrosis of the upper respiratory tract: a mucosal variant of granuloma faciale? A report of three cases. Histopathology 1985;9:1217-25
2 Roberts PF, McCann BG. Eosinophilic angiocentric fibrosis of the upper respiratory tract: a postscript. Histopathology 1997;31:385-6

3 Fageeh NA, Mai KT, Odell PF. Eosinophilic angiocentric fibrosis of the subglottic region of the larynx and upper trachea. J Otolaryngol 1996;25:276-8

4 Loane J, Jaramillo M, Young HA, Kerr KM. Eosinophilic angiocentric fibrosis and Wegener's granulomatosis: a case report and literature review. J Clin Pathol 2001;54:640-1

5 Altemani AM, Pilch BZ, Sakano E, Altemani JM. Eosinophilic angiocentric fibrosis of the nasal cavity. Mod Pathol 1997;10:391-3

6 Burns BV, Roberts PF, De Carpentier J, Zarod AP. Eosinophilic angiocentric fibrosis affecting the nasal cavity. A mucosal variant of the skin lesion granuloma faciale. J Laryngol Otol 2001;115:223-6

7 Matai V, Baer S, Barnes S, Boxer M. Eosinophilic angiocentric fibrosis. J Laryngol Otol 2000;114:563-4

8 Thompson LD, Heffner DK. Sinonasal tract eosinophilic angiocentric fibrosis. A report of three cases. Am J Clin Pathol 2001;115:243-8

9 Holmes DK, Panje WR. Intranasal granuloma faciale. Am J Otolaryngol 1983;4:184-6

10 Pereira EM, Millas I, Reis-Filho JS, Maeda SA, Franco M. Eosinophilic angiocentric fibrosis of the sinonasal tract: Report on the clinicopathologic features of case and review of the literature. Head Neck 2002;24:307-11

11 Tabaee A, Zadeh MH, Proytcheva M, LaBruna A. Eosinophilic angiocentric fibrosis. J Laryngol Otol 2003;117:410-13

12 Nguyen DB, Alex JC, Calhoun B. Eosinophilic angiocentric fibrosis in a patient with nasal obstruction. Ear Nose Throat J 2004;83:183-6

13 Goldner R, Sina B. Granuloma faciale: the role of dapsone and prior irradiation on the cause of the disease. Cutis 1984;33:478-9

14 Van de Kerkhof PC. On the efficacy of dapsone in granuloma faciale. Acta Derm Venereol 1994;74:61-2

15 Marcocci C, Bartalena L, Tanda ML, Manetti L, Dell'Uno E, Rocchi R, et al. Comparison of the effectiveness and tolerability of intravenous or oral glucocorticoids associated with orbital radiotherapy in the management of severe Graves' ophthalmopathy: results of a prospective, single-blind, randomised study. J Clin Endocrinol Metab 2001;86:3562-7

Address for correspondence:

Mr S Paun,

Department of Otolaryngology / Head and Neck Surgery,

St Bartholomew's Hospital,

West Smithfield,

London EC1A 7BE, UK.

E-mail: santdeep.paun@bartsandthelondon.nhs.uk

Mr S Paun takes responsibility for the integrity of the content of the paper.

Competing interests: None declared 